\title{
ESSAIS DE CHIMIOTHERAPIE
}

\section{SUR LA FILAIRE (ICOSIELLA NEGLECTA)}

\section{DE LA GRENOUILLE (RANA ESCULENTA)}

\author{
Par E. LAGRANGE
}

Au cours de l'été 1947, je me suis demandé s'il était possible d'utiliser la grenouille (Rana esculenta) porteuse de filaires (Icosiella neglecta, Diesing, 1851) comme sujet de recherches expérimentales sur la chimiothérapie des filarioses.

L'Icosiella est inconnue en Belgique. Elle semble répandue de façon assez variable en France. J'ai examiné environ 300 grenouilles recueillies dans trois foyers divers.

A Richelieu (Indre-et-Loire) et environs : sur 193 examinées, 30 ont été trouvées infestées, soit $15,50 / 0$.

A La Bazoche-Gouet (Eure-et-Loir) et environs : sur 71 examinées, 25 ont èté trouvées infestées, soit $35,20 / 0$.

A Vannes (Morbihan) : sur une quarantaine de grenouilles, prélevées dans trois gîtes, aucune n'a été trouvée infestée.

Le regretté Camille Desportes, qui m'a aidé dans mes captures aux environs de Richelieu, rapprochait cette dernière constatation des observations qu'il avait faites en Camargue et en Charente ; au voisinage de la mer il n'a jamais trouvé de grenouilles infestées et il supposait que le climat marin écarte la Forcipomyia, dont il a démontré le rôle vecteur dans l'infestation de la grenouille.

La belle saison finie, il m'a été impossible de renouveler mon stock de grenouilles en France. De Léopoldville, j'ai reçu par avion quelques envois. Dans un lot d'une quarantaine de jeunes Rana mascaragnensis, aucune n'était infestée de filaires. Sur un échantillon unique de Rana galamensis. (espèce rare, inconnue jusqu'à présent à Léopoldville) il y avait en abondance deux espèces de microfilaires à gaîne ; à l'autopsie, je n'ai trouvé d'adultes (mâles et femelles) que d'une espèce, pelotonnés la plupart dans deux

Ans, de Parasitologie, T. XXIV, Nos 1-2. - 1949, p. 49-53.

4. 
logettes sous-péritonéales dans les deux hypocondres: je n'ai pu en obtenir la détermination.

Sur 39 Rana occipilalis, trois seulement présentaient des microfilaires à gaîne d'une seule espèce dans le sang. Les microfilaires étaient les mêmes que chez $R$. galamensis.

Après traitement, toutes les grenouilles ont été autopsiées et le sang a été examiné au microscope. Aucun des produits essayés ne semble avoir eu d'action sur les microfilaires. Après examen complet, le cadavre de grenouille était plongé jusqu'au lendemain dans un bocal d'eau physiologique ; de cette façon, les filaires vivantes sortent souvent du cadavre où elles étaient cachées.

Des résultats assez intéressants ont été obtenus avec le violet de méthyle et la dipharsine, qui me paraissent mériter d'ètre rapportés.

Violet de méthyle, - Ce produit, que j'avais essayé in vitro sur les microfilaires de Dirofilaria repens et qui les tue à la concentration d' $1 / 400.000^{\circ}$ en 17 heures, est d'un maniement délicat in vivo sur le chien.

Chez la grenouille, je l'ai administré en injection par voie péri.. tonéale, per os, en lavement et en injection intraveineuse. Les trois premières voies doivent être rejetées. La voie péritonéale, solution à $2 \%$, est inefficace. La voie entérale est dangereuse et grossière. Par contre, la voie intraveineuse est intéressante et efficace.

L'animal étant attaché sur le dos, l'injection se fait, par une boutonnière à la peau, dans une veine de la paroi abdominale, avec du violet de méthyle en solution à $2,5 \%$ neutralisée, à raison de $0,012 \mathrm{~g} . / \mathrm{kg}$. Il convient de faire l'injection lentement pour éviter un choc dangereux.

Trois grenouilles reçoivent du violet de méthyle à raison de $0,15 \mathrm{~cm}^{3}$ pour $40 \mathrm{~g}$., en une seule injection, le 3 septembre. Des essais de toxicité ont prouvé qu'elles supportent sans inconvénient 0,30 et $0,35 \mathrm{~cm}^{3}$, même à doses répétées. Deux sont sacrifiées cinq jours après : chez l'une, pas de filaires adultes (sang + ), chez l'autre, un mâle vivant, une femelle vivante avec évolution des microfilaires arrêtées au stade gastrula (sang + ). Une est sacrifiée 18 jours après (sang + ). On trouve une filaire femelle morte et s'enlevant par lambeaux, alors que les microfilaires sont encore vivantes et grouillantes dans toute la longueur du tube génital, jusqu'à la queue.

J'ai traité aussi quatre grenouilles congolaises : une Rana galamensis de $45 \mathrm{~g}$. reçoit $6 \mathrm{mg}$. au $\mathrm{kg}$. Morte le lendemain, elle montre à l'autopsie 9 filaires femelles et 7 mâles vivants. 
Trois Rana occipitalis de 100 à $135 \mathrm{~g}$. sont traitées au violet de méthyle à $1 \%$ et reçoivent trois injections intraveineuses en huit jours, chacune de $2,6 \mathrm{mg} ! / \mathrm{kg}$. ; la dernière $(100 \mathrm{~g}$.) reçoit $2,5 \mathrm{mg}$. au kilo en une injection. Sacrifiées 18 jours après le début du traitement, je n'y trouve aucune filaire adulte, mais le sang montre encore des microfilaires.

Dipharsine U.C.B. - Le $\mathrm{D}^{r}$ Houssiaux a eu l'obligeance de me signaler au mois d'août 1947 l'amélioration remarquable qu'il avait observée chez un malade, à la suite d'injections répétées de dipharsine (chlorhydrate d'oxyamino-phényl-dichlorarsine). Ce malade, dont le sang n'avait jamais présenté de microfilaires, présentait aux deux poignets des œedèmes filariens (Loa loa) qui le rendaient incapable de tout travail manuel. Le traitement coïncida avec une fonte rapide des oedèmes et retour à la capacité de travail. Le malade se déclare fortement soulagé. Il promet de se représenter si les symptômes réapparaissent. Il n'en fait rien, du reste, et plus tard je l'ai retrouvé, par hasard, se plaignant de ses manifestations filariennes. Devant les résultats momentanément favorables observés, qui semblaient confirmés par les faits tout récemment publiés par Otto et Marren, j'ai traité quelques grenouilles par voie intrapéritonéale.

Essais de toxicité. - A l'U.C.B., la dipharsine est tolérée à raison de $35 \mathrm{mg} . / \mathrm{kg}$. chez le rat (1 injection intraveineuse).

Chez l'homme, le traitement comporte plusieurs injections à $1,4 \mathrm{mg} . / \mathrm{kg}$. Chez la grenouille, j'ai injecté $3,75 \mathrm{mg} . / \mathrm{kg}$. par voie intrapéritonéale, à plusieurs reprises.

Le 15 septembre 1947, six grenouilles filariées reçoivent à quatre reprises (sauf $\mathrm{A}$, à trois reprises) des injections de dipharsine au $1 / 10.000^{\circ}$, calculées à raison de $1,5 \mathrm{~cm}^{3}$ pour 40 g., soit $3,75 \mathrm{mg} . / \mathrm{kg}$. (injections le 15 , le 17 , le 19 et le 23 septembre). Elles sont sacrifiées sucessivement :

- Le 27-9 (sang +++ ). On trouve 2 mâles vivants et 3 femelles, toutes intramusculaires. Une est quasi-morte; après deux petits mouvements elle demeure immobile, pas de microfilaires dans l'utérus. Une morte en lambeaux, pas d'embryons vermiformes; tune morte avec embryons vermiformes immobiles.

- Le 30-9 (échantillon $\mathrm{A}$, après 3 injections) (sang + ). On trouve 2 femelles en lambeaux, avec microfilaires mobiles vermiformes.

- Le 30-9 (sang +). On trouve 5 mâles, dont 2 vivants; une femelle morte, avec embryons vermiformes morts; une femelle morte, en lambeaux, avec embryons morts. 
- Le 6-10 (sang ++ ). On ne trouve pas de mâle; 7 femelles sont dans l'état suivant: une quasi-morte, quelques légers mouvements de la tête ; deux mortes, sans aucun élément mobile ; une en lambeaux, avec des microfilaires mobiles in utero; deux en lambeaux, tous les embryons sont morts; une en partie digérée, montrant par endroits des microfilaires mortes in utero.

- Le 13-10 : les deux dernières grenouilles sont trouvées mortes dans le même aquarium. Une contient cinq femelles et deux mâles, tous morts, à un état de décomposition variable (sang +++ ) ; une contient trois femelles mortes, dont une en lambeaux, pas de microfilaires in utero (sang +++ ).

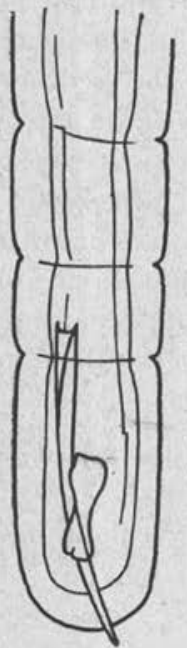

FIG. - Spicules du mâle de la planche V

La dissociation en lambeaux est antérieure à toute manipulation. Il semble que la filaire s'autolyse petit à petit, ne laissant après un certain temps qu'une cuticule, sujette à rupture par les contractions des muscles dans lesquels elles sont glissées. Quand le ver est réduit à une cuticule, il devient évidemment invisible. C'est l'intérêt de la photographie ci-contre, représentant un mâle presque complètement digéré, mais dont les spicules démontrent l'identité.

Voici le bilan d'un autre essai à la dipharsine, par voie intrapéritonéale. Cinq grenouilles de 40 à $50 \mathrm{~g}$. reçoivent successivement une solution au $1 / 10.000^{\circ}$, les 25 août, 28 août, $1^{\text {er }}$ septembre, 4 septembre, $10 \mathrm{~cm}^{3}, 20 \mathrm{~cm}^{3}$ et $40 \mathrm{~cm}^{3}$ au $\mathrm{kg}$. Deux d'entre elles reçoivent encore, le 8 septembre et le 9 septembre, $40 \mathrm{~cm}^{3}$ et $100 \mathrm{~cm}^{3}$ au kg. ; elles sont trouvées mortes le lendemain. Dans l'une d'elles une femelle est morte avec embryons morts; l'autre, une femelle morte, en lambeaux, avec rares microfilaires vivantes dans l'utérus, 2 mâles vivants.

Les autres, sacrifiées respectivement le 8,15 et 24 septembre, contiennent : la première, 1 mâle vivant et 3 femelles mortes, 1 en lambeaux, 2 remplies de microfilaires grouillantes; la deuxième : 2 mâles morts et 2 femelles mortes, 1 en lambeaux, l'autre intacte, avec embryons morts, à développement arrêté ; la troisième : 1 mâle (voir fig.), 1 femelle en lambeaux, avec très rares microfilaires ; le sang de cette grenouille est très richement infesté $(+++)$. Il est donc probable que d'autres parasites m'ont échappé.

Parmi les divers produits que j'ai essayés chez la grenouille, le 
3 
ANNALES DE PARASITOLOGIE

T. XXIV, $N^{\circ s} 1-2,1949$
Planche V

(Mémoire Lagrange)
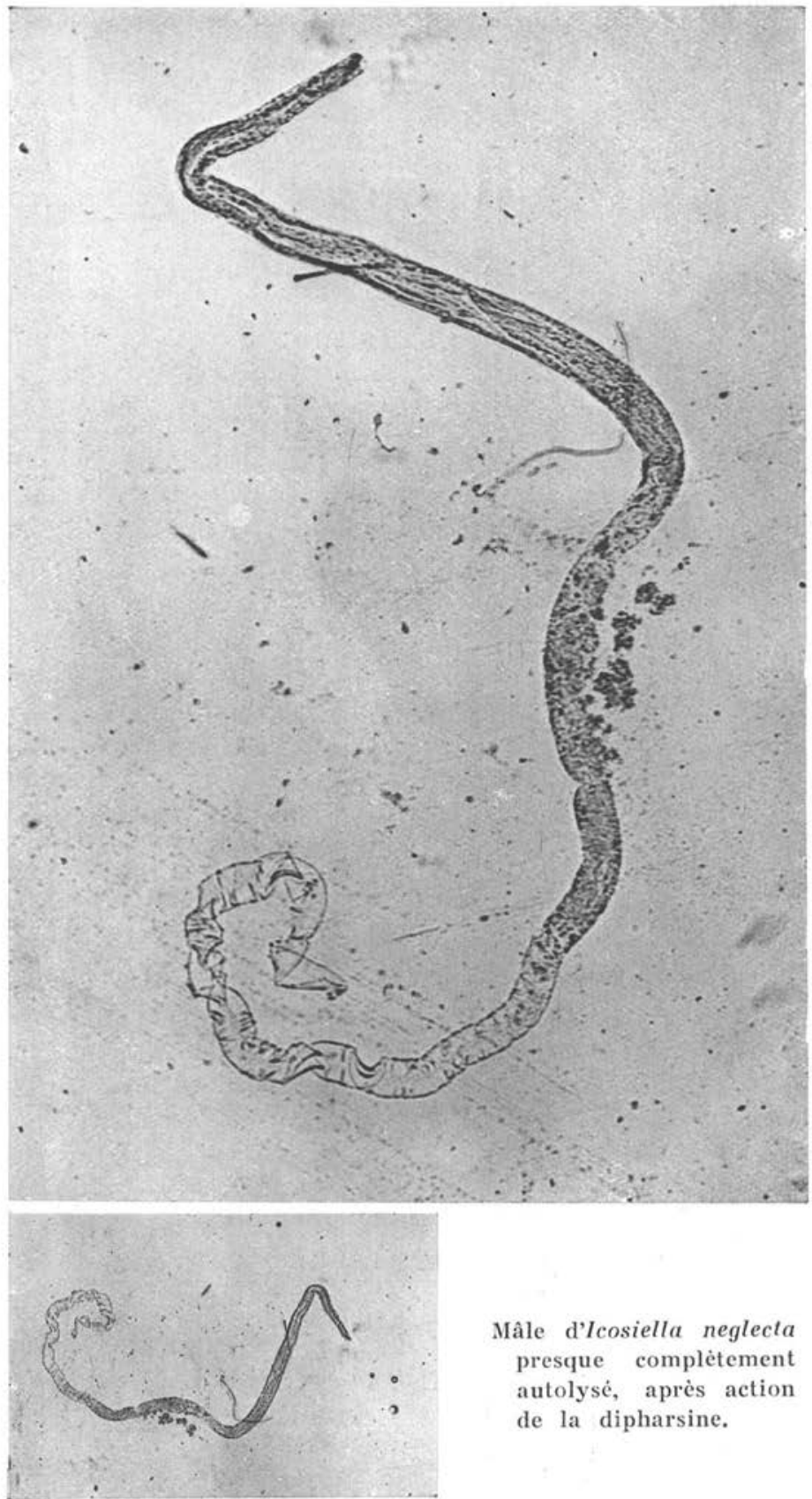

Mâle d'Icosiella neglecta presque complètement autolysé, après action de la dipharsine. 
vluiet de méthyle par voie intraveineuse et la dipharsine (chlorhydrate d'oxyaminophényldichlorarsine) par voie intrapéritonéale m'ont donné des résultats mieux qu'encourageants. Ces résultats sont d'ailleurs beaucoup plus favorables chez la grenouille que chez les mammifères. Le violet de méthyle, dont je donnerai plus tard une étude expérimentale plus poussée, est dangereux chez le rat et le chien : à doses trop élevées, il provoque de l'œedème pulmonaire. Quant à la dipharsine, dans un cas humain et un autre expérimental chez le chien, elle a abouti à un échec.

Si l'expérimentation chez la grenouille offre à coup sûr de grandes facilités, il convient donc d'accepter sous réserves les résultats obtenus chez cet animal.

Je tiens à remercier M. le Pr Brumpt de l'hospitalité qu'il a bien voulu m'accorder dans son laboratoire de Richelieu et à évoquer le souvenir de son regretté collaborateur, Camille Desportes, qui m'a prodigué son aide et ses conseils.

Ce travail a été facilité par une subvention de l'I.R.S.I.A. et de l'Union Chimique Belge, S.A.

Station expérimentale de Richelieu (Indre-et-Loire)

et Laboratoires de Recherches de l'Union Chimique Belge, S.A., Bruxelles 